\title{
Assessment of Molecular Diversity in Chickpea (Cicer arietinum L.) Rhizobia and Structural Analysis of 16S rDNA Sequences from Mesorhizobium ciceri
}

\author{
AKHILESH YADAV ${ }^{1,2}$, ASHA LATA SINGH ${ }^{2}$, GOVIND KUMAR RAI ${ }^{1}$ and MAJOR SINGH*,1 \\ ${ }^{1}$ Division of Crop Improvement, Indian Institute of Vegetable Research, Shahanshahpur, Varanasi, India \\ ${ }^{2}$ Department of Botany, Faculty of Science, Banaras Hindu University, Varanasi, India
}

Submitted 10 January 2013, revised 5 April 2013, accepted 14 June 2013

\begin{abstract}
Molecular diversity studies of 19 rhizobia isolates from chickpea were conducted using simple sequence repeats (SSR) and 16S rDNA-RFLP markers. Phenotypic characterization with special reference to salinity and $\mathrm{pH}$ tolerance was performed. These isolates were identified as different strains of Mesorhizobium, Rhizobium, Bradyrhizobium, and Agrobacterium. Twenty SSR loci of Mesorhizobium ciceri, distributed across the other rhizobial genome, clearly differentiated 19 rhizobial isolates. Analogous clustering supported the results of 16S rDNA sequence-based phylogeny. Analysis of the $16 \mathrm{~S}$ rDNA sequences from M. ciceri strains revealed that nucleotide variables (signature sites) were located at 20 different positions; most of them were present in the first $820 \mathrm{bp}$ region from 5 terminal. Interestingly, 14 signature sites were located in two main regions, the variable region V1 (nt 527-584), and variable region V2 (nt 754-813). The secondary structure and minimal free energy were determined in these two regions. These results will be useful in characterizing the micro-evolutionary mechanisms of species formation and increase understanding of the symbiotic relationship.
\end{abstract}

Ke y w ords: Chickpea, endophyte, genetic diversity, rhizobia, signature site

\section{Introduction}

Chickpea (Cicer arietinum L.; family Leguminoceae) is an important pulse crop valued as a rich source of proteins. Chickpea nodulating rhizobia bacteria are highly host specific (Maatallah et al., 2002) and show distinct cross-inoculation group with respect to nodulation under cross-inoculation experiments (Gaur et al., 1979). Several species of Mesorhizobium, Rhizobium, Agrobacterium, Bradyrhizobium are capable of fixing nitrogen through nodulation in chickpea roots (Maatallah etal., 2002; Bhattacharjee et al., 2008). Under natural field conditions, the nitrogen fixing ability of rhizobia is negatively affected by a variety of stresses including salinity and $\mathrm{pH}$ of soils (Ruiz-Diez et al., 2012). Hence, success of chickpea crop in such soils requires identification of compatible rhizobia, tolerant to variable $\mathrm{pH}$ and osmotic stresses (Ruiz-Diez et al., 2012; Rai et al., 2012).

Based on the coding sequences of small subunit of 16S rRNA, rhizobia belong to either $\alpha$ - or $\beta$-proteobacteria (Moulin et al., 2001). The comparison of rDNA sequences is a powerful tool to deduce phylo- genetic relationships among rhizobia (Alexandre et al., 2006, 2009). Due to crucial structural and functional constraints of the rDNA, they contain highly conserved regions as well as highly variable signatures. Conserved regions proximal to the 5' and 3' termini are present in all prokaryotic $16 \mathrm{~S}$ rDNA sequences thus providing the opportunity to study phylogenetic relationships by comparing the rDNA sequences of different isolates (Alexandre et al., 2006, 2009; Omara et al., 2012). Variations in $16 \mathrm{~S}$ rDNA can also be assessed by analyzing restriction fragment length polymorphism (RFLP) of 16S rDNA sequences (Laguerre et al., 1994; Alexandre et al., 2006; Rai et al., 2012). PCR based markers such as RAPD, RFLP, DAPD and SSR have been used to discriminate bacterial strains and to analyse genetic diversity. (Van Belkum et al., 1998; Alexandre et al., 2006; Laranjo et al., 2002, 2004; Sikora and Redzepovic, 2003; Rai et al., 2012). The availability of the whole genome sequence of Mesorhizobium ciceri has greatly facilitated identification of SSR markers (Van Belkum et al., 1998). In this study, the diversity and structural analysis of fast-growing chickpea rhizobia strains was analysed using SSR, RFLP and partial sequences of $16 \mathrm{~s}$ rDNA.

\footnotetext{
* Corresponding author: M. Singh, Div. of Crop Improvement, Indian Institute of Vegetable Research, P.O. Jakhini, Shahanshahpur, Varanasi-221305, India; phone: +91 9451579735; fax: +91 5443 229007; e-mail: singhvns@gmail.com; akhileshgzp@gmail.com
} 


\section{Experimental}

\section{Materials and Methods}

Isolation and phenotypic characterization of rhizobia. Strains of rhizobia were isolated from root nodules of chickpea plants grown at farmer's field of Mau district (Uttar Pradesh, India) following Vincent (1970) method. The reference strain M. ciceri (Ca181) was procured from CCS Haryana Agriculture University, Hisar, India. Nodulation tests were performed by inoculating chickpea seeds with all the isolates, separately. All isolates including the reference strain were examined for growth at the temperature range from 28 to $30^{\circ} \mathrm{C}, \mathrm{NaCl}$ range from 0.1 to $2.0 \%$ and at three different $\mathrm{pH}$ values (4.5, 7.0, and 9.0) on yeast extract mannitol (YEM) medium. Each experiment was repeated twice with three replications (Ruiz-Diez et al., 2012). Genomic DNA of all the isolates was isolated using GeneiPure ${ }^{\mathrm{TM}}$ bacterial DNA purification kit $\left(\mathrm{GeNe}^{\mathrm{TM}}\right.$, Bangaluru, India) following the manufacture's protocol.

$16 S$ rRNA gene amplification and sequencing. Universal eubacterial primers F-D1-5'-ccgaattcgtcgacaacagagtttgatcctggctcag-3' and R-D1- 5'-cccgggatccaagcttaaggaggtgatccagcc-3' (Kumar et al., 2006) were used to amplify a $1500 \mathrm{bp}$ region of $16 \mathrm{~S}$ rRNA gene using a thermal cycler (BioRad, USA). Amplification products were resolved by electrophoresis in agarose (1\%), and visualised using a gel documentation system (Alfa Imager, Alfa Innotech Corporation, USA). The amplicons were purified using GeneiPure ${ }^{\mathrm{TM}}$ quick PCR purification kit $\left(\mathrm{GeNei}^{\mathrm{TM}}\right.$, Bangaluru, India) and quantified at $260 \mathrm{~nm}$ using a spectrophotometer taking calf thymus DNA as a control. The purified partial $16 \mathrm{~S}$ rDNA amplicon was sequenced in an Applied Biosystems 3130 Genetic Analyzer (Applied Biosystems ${ }^{\oplus}$, CA, USA).

Analysis of $16 \mathrm{~S}$ rDNA sequences. The partial sequences were compared with sequences from DNA databases and sequences showing $>95 \%$ similarity were retrieved by Nucleotide Basic Local Alignment Search Tool (BLAST) program available at the National Center for Biotechnology Information (NCBI) BLAST server (www.ncbi.nlm.nih.gov/BLAST). Sequences were analyzed using the software BIOEDIT (Sequence Alignment Editor ver. 7.0.9; Hall, 1999). The phylogenetic tree was constructed using the software MEGA4 (Molecular Evolutionary Genetics Analysis; Tamura et al., 2007) utilizing Neighbour-Joining method, based on a distance matrix with the distance correction calculated by Kimura's two-parameter model. The secondary structure and minimal free energy were calculated by RNAstructure 5.3 (Reuter et al., 2010). The 16S-rDNA sequences obtained from native isolates were compared with those of rhizobial reference strains available in the GenBank database (Table I).

PCR-RFLP analysis of $16 \mathrm{~S}$ rDNA. For RFLP restriction, PCR amplified products of $16 \mathrm{~S}$ rRNA gene were digested with three tetra-cutting endonucleases, viz., BstUI, HaeIII and MspI (Fermentas). Purified PCR products $(12.3 \mu \mathrm{l})$ were digested with $3 \mathrm{U}$ of restriction endonucleases in a $14 \mu \mathrm{l}$ reaction volume. The digestion products were separated by electrophoresis on agarose gel $1.5 \%$ and the visualized restriction patterns were photographed (Fig. 1). The unweighted pair group

Table I

Reference species type strains and 16S-rDNA accession numbers available in GenBank database for phylogenetic analysis and structure analysis of $16 \mathrm{~S}$ rDNA sequences from strains of $M$. ciceri with other rhizobia

\begin{tabular}{|l|l|l|}
\hline \multicolumn{1}{|c|}{ Species type strain } & $\begin{array}{c}\text { 16S-rDNA } \\
\text { Accession number }\end{array}$ & \multicolumn{1}{c|}{ Reference } \\
\hline Mesorhizobium ciceri strain GA-2 & EF535812.1 & Verma et al. (2010) \\
\hline Mesorhizobium ciceri strain UPM-Ca7 & DQ444456.1 & Terefework et al. (1998) \\
\hline Mesorhizobium ciceri strain Ca181 & GU196798.1 & Goel et al. (2002) \\
\hline Mesorhizobium ciceri strain FCA08 & AY195845.1 & Rivas et al. (2006) \\
\hline Mesorhizobium ciceri strain TS56 & FM209490.1 & Aitouhmane et al. (Unpublished) \\
\hline Mesorhizobium ciceri strain Rcd301 & AY217118.1 & Agrawal et al. (2011) \\
\hline Mesorhizobium ciceri strain C-2/2 & AY206686.1 & Verma et al. (2009) \\
\hline Mesorhizobium ciceri strain Rch9816 & AJ487829.1 & Maatallah et al. (2002) \\
\hline Rhizobium sp. DV2 & DQ873663.1 & Leelahawonge et al. (2009) \\
\hline Rhizobium sp. SWFU-R27 & JN896883.1 & Wang (Unpublished) \\
\hline Agrobacterium tumefaciens strain SWFU-R30 & JN896884.1 & Wang (Unpublished) \\
\hline Agrobacterium tumefaciens strain 23C45 & JN624693.1 & Mnasri et al. (2012) \\
\hline Mesorhizobium sp. CCNWGS0211 & JN622153.1 & Xu (Unpublished) \\
\hline Mesorhizobium ciceri strain 8-2 & JN105987.1 & Halbouni et al. (Unpublished) \\
\hline Bradyrhizobium japonicum strain HHL-01 & DQ517956.1 & Li (Unpublished) \\
\hline Bradyrhizobium japonicum strain CCBAU 83623 & EU145982.1 & Han et al. (Unpublished) \\
\hline
\end{tabular}




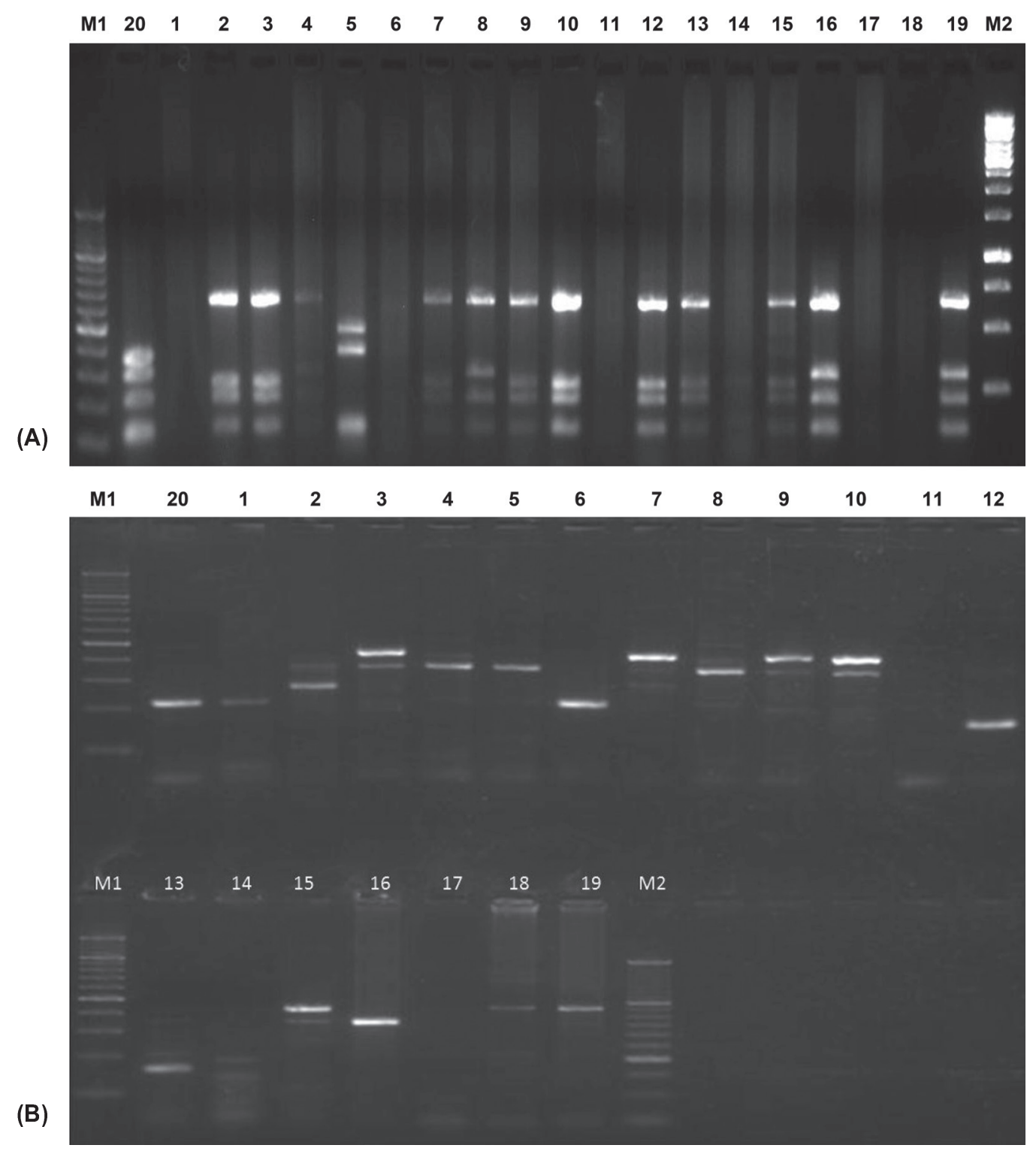

Fig. 1. (A) One of the gel photographs of PCR-RFLP digested with MspI of rhizobial isolates.

(B) One of the gel photographs of SSR profile of rhizobial isolates with primer MSAY-17.

Lanes: M1, 100 bp DNA ladder; M2, 50 bp DNA ladder; 1-20, rhizobial isolates MSA1-20.

method with averages (UPGMA) was used to construct dendogram using the software NTSYS2pc (ver. 2.1).

PCR amplification of simple sequence repeats. A genome-wide search was performed to identify SSR loci in M. ciceri strain using the Tandem Repeat Finder software (ver. 2.0). Non-redundant SSR loci were then selected for designing a total of 20 primers (Table II) using the software Primer 3 (ver. 0.4.0) with a target amplicon size of 150-500 bp. The amplification products were resolved and visualised as described above.

\section{Results}

Phenotype of bacteria. The bacterial colonies produced on YEM agar were gummy, translucent, circular and convex with smooth margins. All the 19 isolates were gram negative and rod-shaped. Majority of the isolates were classified as fast-growing rhizobia, as their colonies reached to a diameter $>2 \mathrm{~mm}$ within $6 \mathrm{~d}$ of growth. The isolates MSA-3, MSA-5, MSA-6, MSA-7, MSA-14 and MSA-20, however, were found to be slowgrowers, as even after $10 \mathrm{~d}$ their colonies could be able to reach to a diameter of $\leq 1 \mathrm{~mm}$. All the isolates were able to grow at the temperature range from 28 to $30^{\circ} \mathrm{C}$ (Table III), which fits with reference $M$. ciceri Ca181 strain. The isolates MSA-3, MSA-5, MSA-6, MSA-7, MSA-14 and MSA-20 showed moderate tolerance to salinity $(1 \% \mathrm{NaCl})$, while the remaining isolates were not able to grow at $0.5 \% \mathrm{NaCl}$. However, M. ciceri $\mathrm{Ca} 181$ showed growth up to $1.5 \% \mathrm{NaCl}$. All the isolates showed vigorous growth at $\mathrm{pH} 7.0$, the optimal $\mathrm{pH}$ for rhizobial growth, and were able to grow at a higher $\mathrm{pH}$ 
TableII

List of SSRs primer used in this study

\begin{tabular}{|l|l|l|}
\hline Primer name & \multicolumn{1}{|c|}{ Forward sequence $\left(5^{\prime}-3^{\prime}\right)$} & \multicolumn{1}{c|}{ Reverse sequence ( ${ }^{\prime}$ - $\left.3^{\prime}\right)$} \\
\hline MSAY-01 & ACTGTCTCGCTGTCGCTGT & GTTTACGGCGAGAAGGTTGA \\
\hline MSAY-02 & GATGCCACCTGGTCGAAG & CGAAATAGGCGCAGGAATAC \\
\hline MSAY-03 & GAGCGGCATTCCCTCTTT & GATGCCATCCTGCGTCTT \\
\hline MSAY-04 & TATTAAGACGCCGCGAAAGT & ATGACGAGGACGATGTGGAC \\
\hline MSAY-05 & GCCGATGCTCCACCATTC & GAGATGATCGCCCGCAAG \\
\hline MSAY-06 & GATCGATCTCGAGGTAAAAGC & TTCCGATCTAAGCAGAAGACG \\
\hline MSAY-07 & TCGGAAAAGACCGTCACTCT & TTCAAGCAGAAGACGGCATA \\
\hline MSAY-08 & CTGGGTCCTGCGTCAAAG & ACCGTGTCGAAAAGGACATC \\
\hline MSAY-09 & ATTCGTTGGCGAGTTCTACC & ACCGAGTTCAAGCTGGAGAG \\
\hline MSAY-10 & GGACCAGGCTGGACAAAATA & GAGCCTGGCATGATCGAA \\
\hline MSAY-11 & CCTTATGGCGTCCCATACC & TCCCTTGTTTAACCGCTGTC \\
\hline MSAY-12 & GGACGGAGACAATGACCATC & GTATTGGCGACCACGAACTC \\
\hline MSAY-13 & GCATTTTCGGCGATTTAGC & TGCCATACCGAATCTGTTCA \\
\hline MSAY-14 & TGAAAAGCTTGCACATCTCG & TTTACGGCGAGAAGGTTGAC \\
\hline MSAY-15 & GTGGCCGTTCTGGTAGATGT & CGCGCCAATATTCAGTACG \\
\hline MSAY-16 & GGACGGCGAACTCGATATAC & TAGGCGCAGGAATACTGCAT \\
\hline MSAY-17 & CAAGAACGCATTCCTGACG & GTTCGGCAATAAAGGACAGG \\
\hline MSAY-18 & GCTCCTGGTCAAAAAGATCG & GAGTTCTACCGTGCCTTCCA \\
\hline MSAY-19 & TTTAAATTGACGGGGACAGC & GTATTGGCGACCACGAACTC \\
\hline MSAY-20 & CGCTTATAGTTGCGTTGCAC & TGCCATACCGAATCTGTTCA \\
\hline
\end{tabular}

Table III

Survival of isolates obtained from root nodules and reference rhizobial strain under, $\mathrm{pH}$ and salinity tolerance

\begin{tabular}{|l|c|c|c|c|c|c|}
\hline \multirow{2}{*}{ Strain } & \multicolumn{7}{|c|}{ Salt tolerance $\mathrm{a}(\mathrm{NaCl} \%)$} & \multirow{2}{*}{$\begin{array}{c}\text { pH range } \\
4.5-9.0\end{array}$} \\
\cline { 2 - 7 } & 0.1 & 0.5 & 1.0 & 1.5 & 2.0 & $7-9$ \\
\hline MSA-3, 5, 6, 7, 14, 20 & +++ & ++ & + & + & - & $4.5-9$ \\
\hline MSA-11, 13, 15, 17 & ++ & + & + & - & - & $7-9$ \\
\hline MSA-4, 8, 9, 10, 16, 18, 19 & +++ & - & - & - & - & $7-9$ \\
\hline MSA-1, 2, 12 & +++ & + & + & - & - & \\
\hline Reference strain & \multicolumn{7}{|l|}{} \\
\hline Mesorhizobium ciceri Ca181 & +++ & ++ & ++ & + & - & $7-9$ \\
\hline
\end{tabular}

${ }^{a}$ Growth was represented as -, no growth; +, weak growth (10-30\% compared to the control, YEM medium); + +, good growth (40-80\% compared to the control); +++ , very good growth (equal to the control). (Values represent the average of two experiments with three replicates each time).

value (9.0). The isolates MSA-11, MSA-13, MSA-15 and MSA-17 were found to be resistant to acidity, as they could able to grow at $\mathrm{pH} 4.5$.

PCR-RFLP analysis of $16 \mathrm{~S}$ rDNA amplicons. Analysis of the genetic relatedness among the 19 isolates was done employing amplified rDNA restriction analysis (ARDRA) of $16 \mathrm{~S}$ rRNA gene. A combination of three endonucleases (BstUI, HaeIII, and MspI) permitted a good resolution level. Restriction analysis of $16 \mathrm{~S}$ rDNA with these enzymes resulted in 3 to 4 different patterns for each enzyme. The restriction digestion products ranged from 200 to $1000 \mathrm{bp}$. Based on the restriction fragments, the 19 chickpea rhizobia isolates were grouped into three clusters: Group I, Group II, and Group III (Fig. 2). The reference strain MSA-20 (M. ciceri Ca181) clustered with Group I (GI), and was distinct from the isolates of other clusters. Isolates MSA-2, MSA-3, MSA-7, MSA-8, MSA-9, MSA-10, MSA-12, MSA-13, MSA-15, MSA-16 and MSA-19 showed identical ARDRA pattern with the reference strain M. ciceri Ca181. ARDRA pattern of isolates MSA-1, 5, 4, 14 and MSA-6, 11, 17, 18 were identical respectively. MspI and BstUI produced comparable patterns for MSA-5 and MSA-20. On the basis of ARDRA pat- 


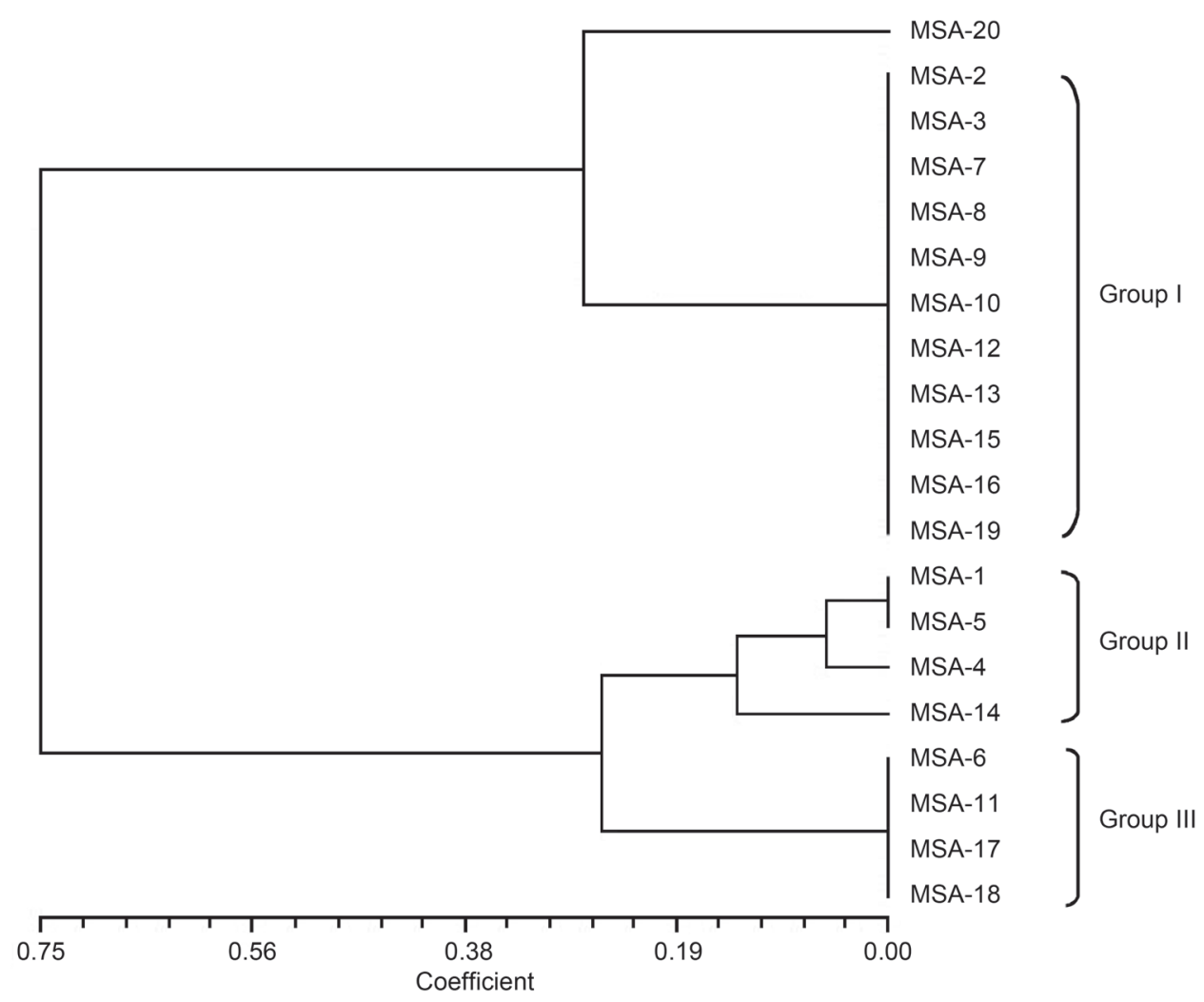

Fig. 2. Unweighted paired-group method using arithmetic averages (UPGMA) cluster of nodule isolates on the basis of 16S ARDRA with HaeIII, MspI and BstUI.

tern four genotypes were detected among the nineteen isolates (Table IV).

Phylogenetic analysis. All the 19 isolates fell into three major phylogenetic groups (Fig. 3). Sequence analysis of $16 \mathrm{~S} \mathrm{rDNA}$ indicated that five isolates had similarity with Rhizobium sp. DV2, four isolates had similarity with M.ciceri strain 8-2, three isolates had similarity with Agrobacterium tumefaciens strain 23C45, four isolates shared maximum similarity with Bradyrhizobium japonicum strain HHL-01, two isolates had similarity with Rhizobium sp. SWFU-R27 and four isolates were classified as Mesorhizobium similarity with Mesorhizobium sp. CCNWGS0211.

Partial 16S rDNA sequences of isolates MSA-5, MSA-6, MSA-14, MSA-20 (M. ciceri-like isolates), isolates MSA-11, MSA-13, MSA-15 MSA-17 (B. japonicum-like isolates), isolates MSA-4, MSA-8, MSA-9. MSA-10, MSA-16, MSA-18, MSA-19 (Rhizobium sp.like isolates), isolates MSA-3, MSA-7 (Mesorhizobiumlike isolates), and isolates MSA-1, MSA-2, MSA-12 (A.tumefaciens-like isolates) were used to construct phylogenetic tree (Fig. 3). The MSA sequences were compared with 6 rhizobial isolates belonging to different species. Phylogenetic analyses indicated that isolates belonging to the $M$. ciceri group were in the same ancestral clade with M. ciceri 8-2 (99-100\% bootstrap probability), whereas isolates belonging to Mesorhizobium group were intermingled with strain Mesorhizobium sp.
Table IV

Rhizobial isolates of chickpea and reference strain used in this study and results from $16 \mathrm{~S}$ rDNA ARDRA

\begin{tabular}{|l|c|}
\hline \multicolumn{1}{|c|}{ Strain/species } & ARDRA genotype ${ }^{\text {a }}$ \\
\hline MSA-1 & 3 \\
\hline MSA-2 & 1 \\
\hline MSA-3 & 3 \\
\hline MSA-4 & 3 \\
\hline MSA-5 & 2 \\
\hline MSA-6 & 1 \\
\hline MSA-7 & 1 \\
\hline MSA-8 & 1 \\
\hline MSA-9 & 1 \\
\hline MSA-10 & 2 \\
\hline MSA-11 & 1 \\
\hline MSA-12 & 1 \\
\hline MSA-13 & 4 \\
\hline MSA-14 & 1 \\
\hline MSA-15 & 1 \\
\hline MSA-16 & 2 \\
\hline MSA-17 & 2 \\
\hline MSA-18 & 1 \\
\hline MSA-19 & 4 \\
\hline MSA-20 (Reference Strain) & 1 \\
\hline Mecif & 1 \\
\hline
\end{tabular}

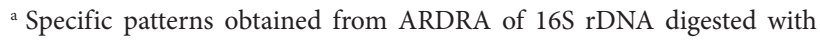
endonucleases respectively Different numbers were assigned to represent each ARDRA group. 


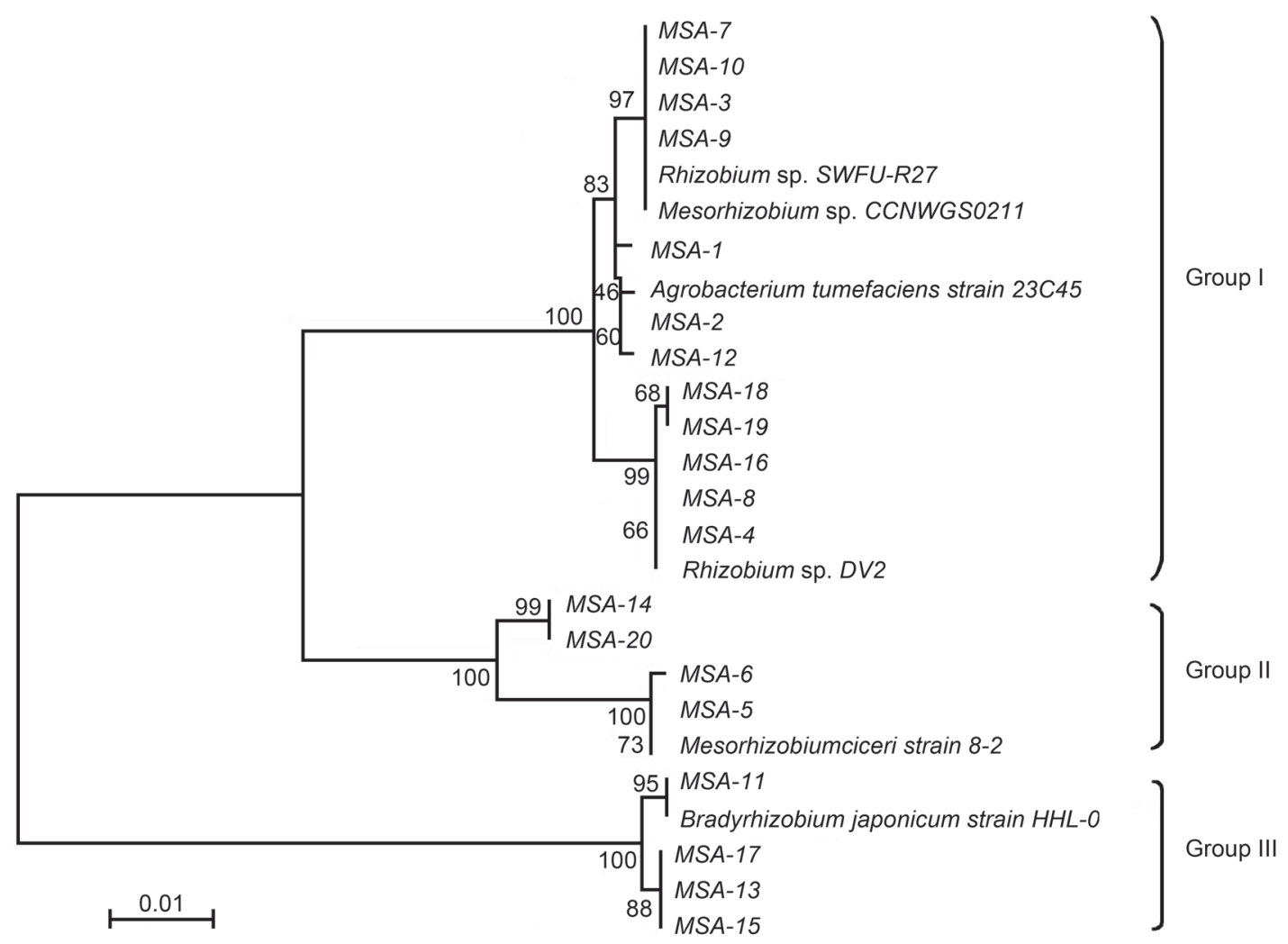

Fig. 3. Phylogenetic relationship between rhizobial isolates based on partial length $16 \mathrm{~S}$ rDNA sequences constructed using cluster algorithm.

CCNWGS0211 (97\% bootstrap probably; Fig. 3). Two Rhizobium-like isolates (MSA-9 and MSA-10) were in the same clade with Rhizobium sp. SWFU-R27 (97\% bootstrap probability). The isolates MSA-1, MSA-2 and MSA-12 that were identified as Agrobacterium strain, shared the same clade as A. tumefaciens (60\% bootstrap probability). Isolates MSA-4, MSA-8, MSA-16, MSA-18 and MSA-19 formed genetic clusters with Rhizobium sp. DV2 (66-68\% bootstrap probability), while isolates MSA-11, MSA-13, MSA-15 and MSA-17, formed genetic clusters with B. japonicum (88-95\% bootstrap probability). High bootstrap values in the phylogenetic study established that the sequenced isolates were properly clustered in the same branches so as the respective reference strains. These phylogenetic relationships allowed a more accurate description when compared with ARDRA technique for the genetic affiliation of chickpea rhizobial isolates.

Simple sequence repeats (SSRs) analysis. Twenty SSR primers were capable of differentiating the rhizobial isolates. The amplification products ranged from $150 \mathrm{bp}$ to $500 \mathrm{bp}$. Despite the polymorphism observed with several primers, the diversity index was low. Cluster analysis based on SSRs revealed that the 19 isolates grouped into four major clusters (Fig. 4). The rhizobial isolates MSA-1, MSA-6, MSA-12, MSA-20 clustered together to form group I, isolates MSA-4, MSA-5, MSA-8, MSA-16, MSA-18, MSA-19 formed group II, isolates MSA-3, MSA-7, MSA-9, MSA-10, MSA-15 clustered to group III, and MSA-11, MSA-13, MSA-14, MSA-17 formed group IV. The isolates MSA-2 formed a separate independent lineage on dendrogram but have similarity with group II. The reference strain MSA20 formed cluster with group I, and showed a similarity with MSA-1 and MSA-6.The results indicated that SSR provided a high degree of discrimination between the strains.

Structural analysis of $16 \mathrm{~S}$ rDNA sequences. The complete alignment of 16S-rDNA sequences of four $M$. ciceri sequences with reference strains available in database (Table I) showed that the intra-specific variations (signature sites) were not randomly distributed (Table V and supplementary material). Nucleotide variables were detected at 20 different positions and most of them were in the first $820 \mathrm{bp}$ region from 5 ' terminal. Interestingly, the first 14 signature sites were located in two main regions, variable region V1 (nt 527-584), and variable region V2 (nt 754-813). The secondary structure and minimal free energy of these two main regions were calculated by RNA structure 5.3 (Kulkarni and Nautiyal, 1999). To simplify the process, M. ciceri strain C-2/2 and M. ciceri strain Ca181 were used as the representation of group I and group II, respectively (Fig. 5). The results revealed that the structures of strain C-2/2 and Ca181 were almost similar with different minimal free energy (Fig. 6A) for variable region V1 


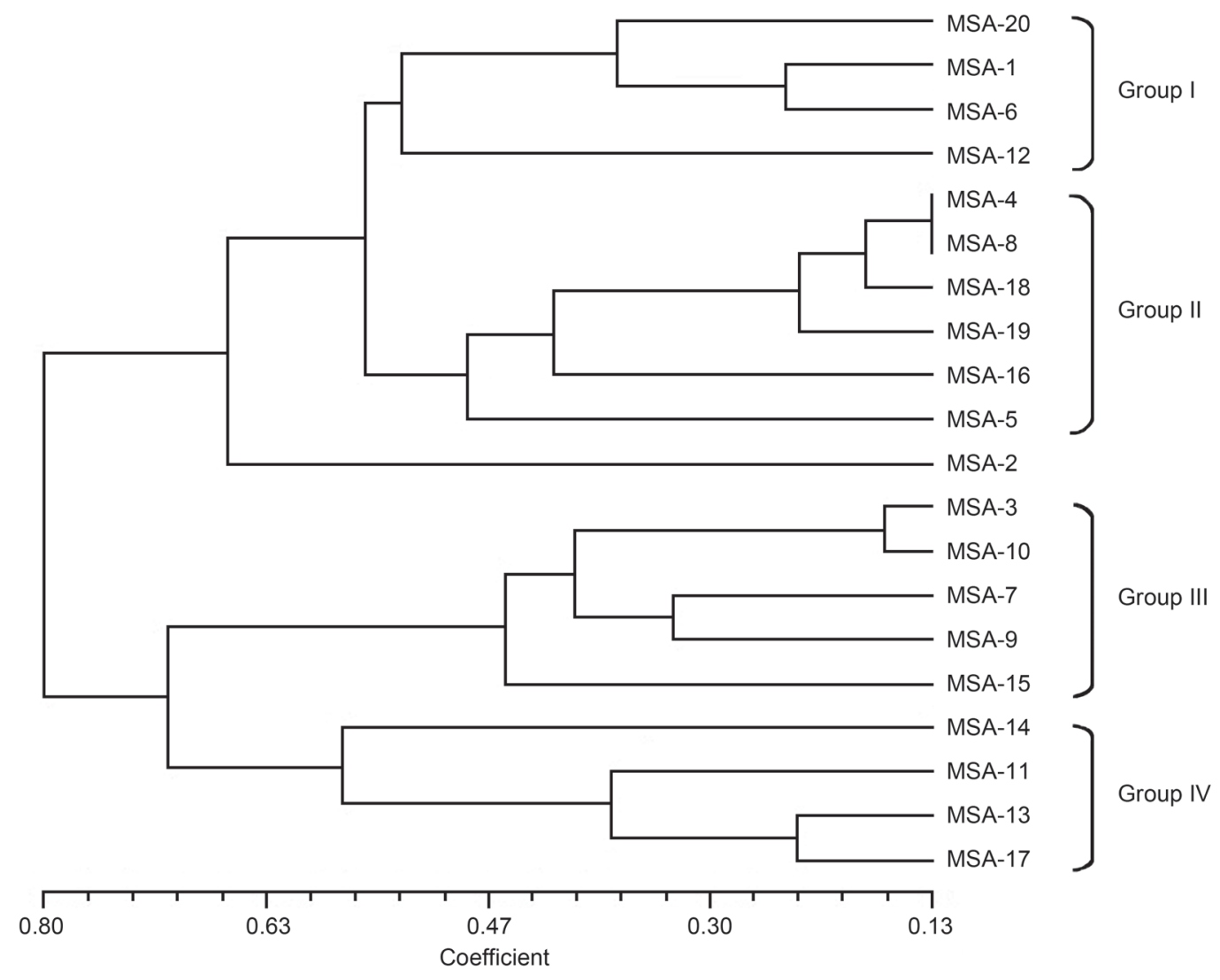

Fig. 4. Dendrogram of genetic similarity among rhizobial isolates based on UPGMA cluster analysis of data from twenty SSR loci.

Table $\mathrm{V}$

Signature Sites for two groups

\begin{tabular}{|l|c|c|}
\hline \multicolumn{1}{|c|}{ Position $^{*}$} & Group I & Group II \\
\hline 342 & C & A \\
\hline 527 & A & T \\
\hline 528 & T & G \\
\hline 535 & C & T \\
\hline 550 & G & A \\
\hline 562 & C & A \\
\hline 575 & G & C \\
\hline 583 & G & A \\
\hline 584 & T & T \\
\hline 754 & G & A \\
\hline 758 & G & G \\
\hline 759 & A & C \\
\hline 770 & T & C \\
\hline 812 & T & T \\
\hline 813 & C & T \\
\hline 945 & C & T \\
\hline 958 & G & A \\
\hline 1075 & G & C \\
\hline 1229 & & C \\
\hline 1415 & C & T \\
\hline
\end{tabular}

* using the type strain M. ciceri UPM-Ca7 16S rDNA sequences numbering convention (nt 527-584). For variable region V2 (nt 754-813), however, the structure and minimal free energy were different (Fig. 6B).

\section{Discussion}

In the present study, all the isolates were shown to be gram-negative, aerobic, non-spore forming cocci. Colony morphology of rhizobia plays an important role in symbiotic nitrogen fixation (Vincent, 1970). All rhizobial isolates were able to form nodules in chickpea roots (Table VI); the nodulation ability did not vary among the groups. Although, SSR and ARDRA approaches provided some degree of information on the diversity, it was not enough to demonstrate the large diversity found amongst the isolates. The diversity in $16 \mathrm{~S}$ rDNA

Table VI

Plant nodulation test assays of different rhizobial isolates

\begin{tabular}{|l|c|}
\hline \multicolumn{1}{|c|}{ Strain } & \multicolumn{1}{|c|}{$\begin{array}{c}\text { No. of Average Root } \\
\text { Nodules/plant }\end{array}$} \\
\hline MSA-3, 5, 6, 7, 14, 20 & $13 \pm 2$ \\
\hline MSA-11, 13, 15, 17 & $2 \pm 1$ \\
\hline MSA-4, 8, 9, 10, 16, 18, 19 & $10 \pm 2$ \\
\hline MSA-1, 2, 12 & $2 \pm 1$ \\
\hline Reference strain & $18 \pm 2$ \\
\hline Mesorhizobium ciceri Ca181 & \\
\hline
\end{tabular}




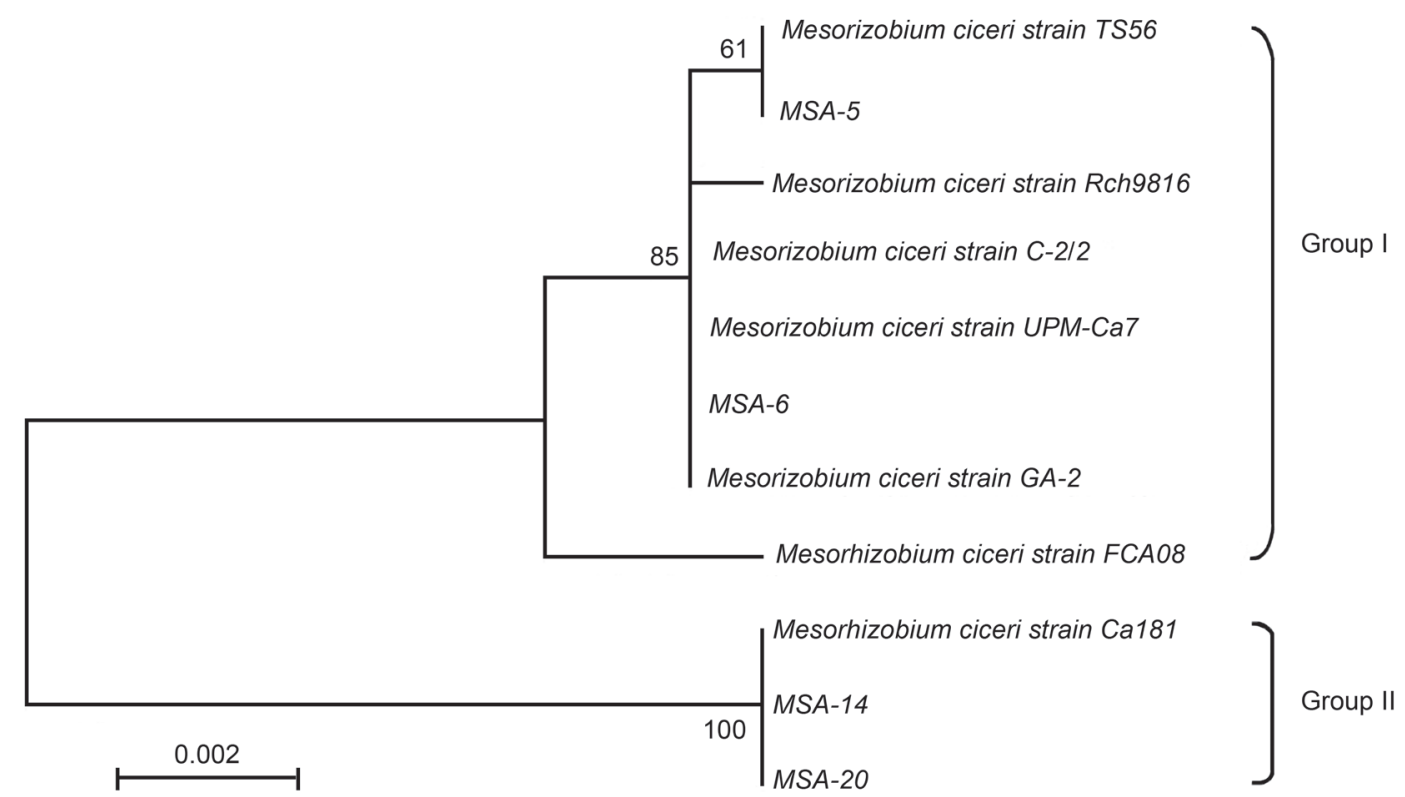

Fig. 5. Phylogenetic tree of various Mesorhizobium ciceri strains based on 16S rDNA sequences for structure analysis.

A

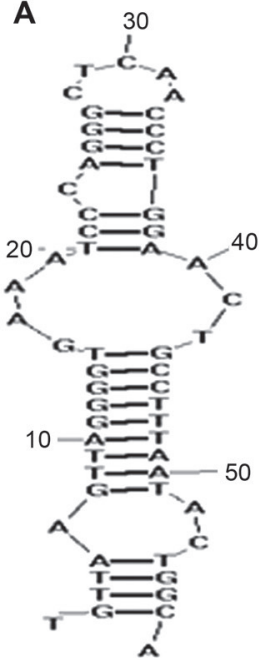

M. ciceri strain C-2/2

Energy $=-22.4$

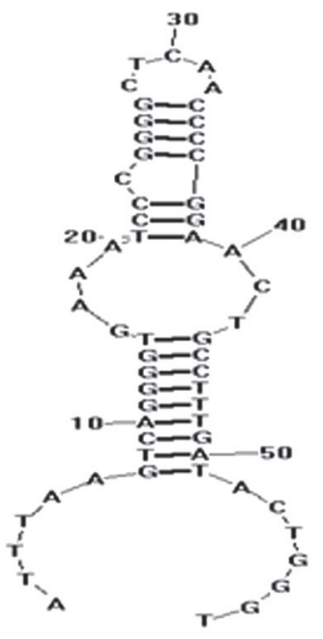

M. ciceri strain Ca181 Energy $=-22.4$

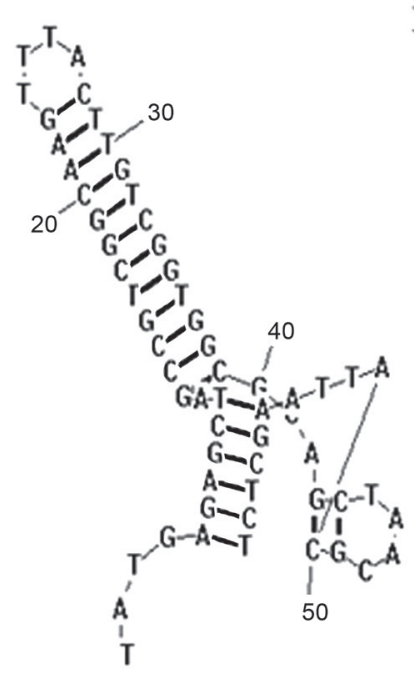

M. ciceri strain $\mathrm{C}-2 / 2$ Energy $=-20.8$

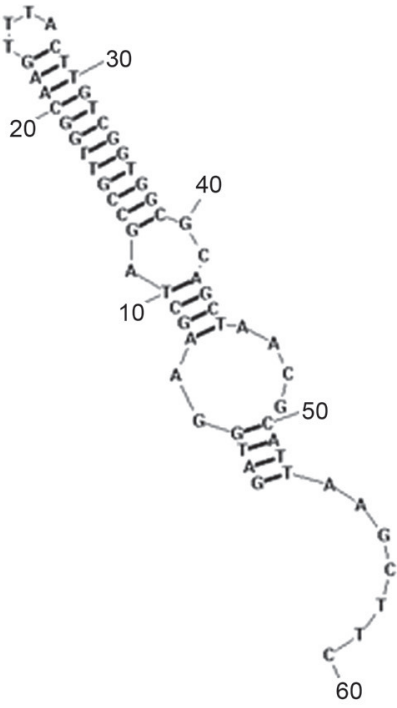

M. ciceri strain Ca181 Energy $=-23.8$

Fig. 6. (A) Secondary structure of the nt 527-584 region of (B) Secondary structure of the nt 754-813 region of $16 \mathrm{~S}$ rDNA for two groups.

sequences among the differnt isolates is a useful method to assess their phylogenetic relationship (Laguerre et al., 1994). Salt tolerance screening revealed that isolates can tolerate $1 \% \mathrm{NaCl}$ and could be potential isolates for salt affected soils. The tolerance to extreme $\mathrm{pH}$ was generally homogeneous between the isolates. The capacity of isolates MSA-11, MSA-13, MSA-15 and MSA-17 to tolerate $\mathrm{pH} 4.5$ was generally low. This could be a feature of growth in culture medium compared to soil where the charges of colloidal particles can partially neutralize the acidity. A lack of correlation between rhizobial growth in acidic soils and pure buffered media has been reported (Kulkarni and Nautiyal, 1999; Hungari et al.,
2001; Ruiz-Diez et al., 2009, 2012). Most of the isolates showed a good growth at $\mathrm{pH}$ 7.0-9.0, a characteristic feature of rhizobia isolated from soybean in Brazil (Yates et al., 2004) and fast-growing rhizobia isolated in Australia (Chen et al., 2005).

Phylogenetic inference, as an approach to establish bacterial relationships is usually based on the comparative analysis of $16 \mathrm{~S}$ rDNA sequences and has previously been used in many investigations (Chen et al., 2005; de Lajudie et al., 1994). The 16S rDNA analysis placed individual rhizobial isolates to three different groups (I to III). Group III comprised of four isolates from Bradyrhizobium. Groups II represented entirely by 
known Mesorhizobium sp. The clade formed by isolates in the group I were from Mesorhizobium, Rhizobium, and Agrobacterium. After sequencing analyes, 17 isolates were identified as three different species of Rhizobium and 3 isolates were identified as Agrobacterium. Lateral gene transfers or mutations may be a cause of these diversions from ARDRA results. Lateral gene transfer dynamics is well known in the populations of free-living soil bacteria (Ueda et al., 1999; Herrera-Cervera et al., 1999). In this study, polymorphism was observed with the combination of three endonucleases (Bst UI, MspI, and HaeIII) and it permitted a resolution level comparable to that reported by Laguerre et al. (1994).

Genomic DNA fingerprinting using SSR was found to be useful in differentiating closely-related isolates. The clustering of the isolates in three groups implies that the chickpea rhizobial isolates were diverse (Fig. 4). The results also indicated that SSR is an efficient method for differentiating and studying diversity and population structure of rhizobia. In addition, this marker system could easily be converted into a multiplex PCR. The signature site in $16 \mathrm{~S}$ rDNA sequences has previously been used as marker to distinguish the isolates at subspecies level (Bertil et al., 1998). Twenty signature sites of $M$. ciceri were identified and most of the variations were detected in the first $820 \mathrm{bp}$ fragment from 5 '-terminal. Interestingly, at variable region V1 (nt 527-584), the structure of group I was similar to group II, which was consistent with the results of phylogenetic tree where both groups had high similarity (Fig. 5). For variable region V2 (nt 754-813), group II had more G/C variations than group I. This may be the reason for a lower minimal free energy of the variable region V2. These signature sites can be used as markers to distinguish the sub-species of $M$. ciceri. The $700 \mathrm{bp}$ fragments from 3'-terminal were highly conserved across the isolates, thus can be used as tool to identify M. ciceri species from other species of Mesorhizobium.

Overall, diversity analysis generated by the different molecular approaches revealed species groups differentiation in chickpea rhizobia isolates. Moreover, structure analysis of $16 \mathrm{~S} \mathrm{rDNA}$ sequences proved to be a quicker and reliable method to differentiate the isolates. The molecular and phenotypic characterization of Indian isolates of rhizobia has advance the knowledge and understanding of chickpea rhizobia isolates, which can help in designing improved production of chickpea in India.

\section{Acknowledgements}

The financial support from Indian Council of Agricultural Research (ICAR), New Delhi in the form of research project 'Application of Microorganism in Agricultural and Allied Sectors (AMAAS)' is gratefully acknowledged. The authors are grateful to Dr. Sanjeev Kumar, Indian Institute of Vegetable Research, Varanasi, India and Dr. Sanjeet Kumar, AVRDC-The World Vegetable Center, Taiwan for valuable suggestions and critical editing.

\section{Literature}

Agrawal P., S. Kumar, Y.K. Jaiswal and H.R. Das. 2011. A Mesorhizobium lipopolysaccharide (LPS) specific lectin (CRL) from the roots of nodulating host plant, Cicer arietinum. Biochimie 93: $440-449$.

Alexandre A., C. Brigido, M. Laranjo, S. Rodrigues and S. Oliveira. 2009. Survey of chickpea rhizobia diversity in Portugal reveals the predominance of species distinct from Mesorhizobium ciceri and Mesorhizobium mediterraneum. Microb. Ecol. 58: 930-941.

Alexandre A., M. Laranjo and S. Oliveira. 2006. Natural populations of chickpea rhizobia evaluated by antibiotic resistance profiles and molecular methods. Microb. Ecol. 51: 128-136.

Bertil P., B. Goran, T. Francois, U. Mathias and J. Karlerik. 1998. Molecular evolution of Mycoplasma capricolum subsp. capripneumoniae strains based on polymorphisms in the 16S rRNA genes. J. Bacteriol. 180: 2350-2358.

Bhattacharjee R.B., A. Singh and S.N. Mukhopadhyay. 2008. Use of nitrogen-fixing bacteria as biofertiliser for non-legumes: prospects and challenges. Appl. Microbiol. Biotechnol. 80: 199-209.

Chen W.X., E.T. Wang and L.D. Kuykendall. 2005. Bergey's manual of systematic bacteriology, $2^{\text {nd }}$ ed., vol. 2, in press. Springer-Verlag, New York, N.Y.

De Lajudie P., A. Willems and B. Pot. 1994. Polyphasic taxonomy of rhizobia: emendation of the genus Sinorhizobium and description of Sinorhizobium meliloti comb. nov., Sinorhizobium saheli sp. nov. and Sinorhizobium teranga sp. nov. Intl. J. Syst. Bacteriol. 44: 715-733. Gaur Y.D. and A.N. Sen. 1979. Cross inoculation group specificity in Cicer-rhizobium symbiosis. New Phytol. 83: 745-754.

Goel A.K., S.S. Sindhu and K.R. Dadarwal. 2002. Stimulation of nodulation and plant growth of chickpea (Cicer arietinum L.) by Pseudomonas spp. antagonistic to fungal pathogens. Biol. Fertil. Soil. 36: 391-396.

Hall T.A. 1999. BIOEDIT: A user-friendly biological sequence alignment editor and analysis program for Window 95/98/NT. Nucl. Acids Symp. 41: 95-98.

Herrera-Cervera J.A., J. Caballero-Mellado, G. Laguerre, H.V. Tichy, N. Requena, N. Amarger, E. Martinez-Romero, J. Olivares and J. Sajuan. 1999. At least five rhizobial species nodulate Phaseolus vulgaris in a Spanish soil. FEMS Microbiol. Ecol. 30: 87-97.

Kulkarni S. and C.S. Nautiyal. 1999. Characterization of high tolerant rhizobia isolated from Prosopis juliflora grown in alkaline soil. J. Gen. Appl. Microbiol. 45: 213-220.

Kumar B., M.S. Kumar, K. Annapurna and D.K. Maheshwari. 2006. Genetic diversity of plant growth-promoting rhizobia isolated from a medicinal legume, Mucuna pruriens Linn. Curr. Sci. 91: 1524-1529.

Laguerre G., M.R. Allard, F. Revoy and N. Amarger. 1994. Rapid Identification of rhizobia by restriction fragment length polymorphism analysis of PCR-amplified 16S rRNA genes. Appl. Environ. Microbiol. 60: 56-63.

Laranjo M., C. Branco, R. Soares, L. Alho, M.D.E. Carvalho and S. Oliveira. 2002. Comparison of chickpea rhizobia isolates from diverse Portuguese natural populations based on symbiotic effectiveness and DNA fingerprint. J. Appl. Microbiol. 92: 1043-1050.

Laranjo M.J. Machado, J.P.W. Young and S. Oliveira. 2004. High diversity of chickpea Mesorhizobium species isolated in a Portuguese agricultural region. FEMS Microbiol. Ecol. 48: 101-107.

Leelahawonge C., N. Pongsilp and A.N. Thammasat. 2009. Factors influencing indole-3-acetic acid biosynthesis of root-nodule bacteria isolated from various leguminous plants. Intl. J. Food Sci. Tech. 14. Maatallah J., E.B. Berraho, S. Munoz, J. Sanjuan and C. Lluch. 2002. Phenotypic and molecular characterization of chickpea rhizobia isolated from different areas of Morocco. J. Appl. Microbiol. 93: 531-540. 
Mnasri B., S. Saidi, S.A. Chihaoui and R. Mhamdi. 2012. Sinorhizobium americanum symbiovar mediterranense is a predominant symbiont that nodulates and fixes nitrogen with common bean (Phaseolus vulgaris L.) in a Northern Tunisian field. Syst. Appl. Microbiol. 35: 263-269.

Moulin L., A. Muniue, B. Dreyfus and C.B. Masson. 2001. Nodulation of legumes by members of the $\beta$-subclass of Proteobacteria. Nature 411: 948-950.

Omara J., T. P. Ekeya and J. F. Hawumba. 2012. Phylogenetic analysis based on 16S rRNA gene of a thermophilic protease-secreting Bacillus gelatini-TPNK-3 isolate from Kiteezi landfill, Uganda. Pol. J. Microbiol. 61: 227-231.

Rai R., P.K. Dash, T. Mohapatra and A. Singh. 2012. Phenotypic and molecular characterization of indigenous rhizobia nodulating chickpea in India. Indian J. Exp. Biol. 50: 340-350.

Reuter S. and D.H. Mathews. 2010. RNAstructure: software for RNA secondary structure prediction and analysis. BMC Bioinformatics 11: 129.

Rivas R., A. Peix, P.F. Mateos, M.E. Trujillo, E. Martinez-Molina, and E. Velazquez. 2006. Biodiversity of populations of phosphate solubilizing rhizobia that nodulates chickpea in different Spanish soils. Plant Soil 287: 23-33.

Ruiz-Diez B., S. Fajardo, M. delRosario deFelipe and M. Fernandez-Pascual. 2012. Characterization of rhizobia from legumes of agronomic interest grown in semi-arid areas of Central Spain relates genetic differences to soil properties. J. Basic Microbiol. 52: 66-78. Ruiz-Diez B., S. Fajardo, M.A. Puertas-Mejia, R. deFelipe and M. Fernandez-Pascual. 2009. Stress tolerance, genetic analysis and symbiotic properties of root-nodulating bacteria isolated from
Mediterranean leguminous shrubs in central Spain. Arch. Microbiol. 191: 35-46.

Sikora S. and S. Redzepovic. 2003. Genotypic characterization of indigenous soybean rhizobia by PCR-RFLP of $16 \mathrm{~S}$ rDNA, rep-PCR and RAPD analysis. Food Tech. Biotechnol. 41: 61-67.

Tamura K., J. Dudley, M. Nei and S. Kumar. 2007. MEGA4: Molecular Evolutionary Genetics Analysis (MEGA) software version 4.0. Mol. Biol. Evol. 24: 1596-1599.

Terefework Z., G. Nick, S. Suomalainen, L. Padin and K. Lindstrm. 1998. Phylogeny of Rhizobium galegae with respect to other rhizobia and agrobacteria. Intl. J. Syst. Bacteriol. 48: 349-356.

Ueda K., T. Seki, T. Kudo, T. Yoshida and M. Kataoka. 1999. Two distinct mechanisms cause the heterogeneity of $16 \mathrm{~S}$ rRNA. J. Bacteriol. 181: 78-82.

Van Belkum A., S. Scherer, L. VanAlphen and H. Verbrugh. 1998. Short sequence DNA repeats in prokaryotic genomes. Microbiol. Mol. Biol. Rev. 62: 275-293.

Verma J.P., J. Yadav, Lavkush, K.N. Tiwari, V., Singh. 2010. Impact of plant growth promoting rhizobacteria on crop production. Intl. J. Agric. Res. 5: 954-983.

Verma J.P., J. Yadav and K.N. Tiwari. 2009. Effect of Mesorhizobium and plant growth promoting rhizobacteria on nodulation and yield of chickpea. Biol. Forum 1: 11-14.

Vincent, J.M. 1970. A Manual for the Practical Study of Root Nodule Bacteria. Blackwell Scientific, Oxford.

Yates R.J., J.G. Howieson, K.G. Nandasena and G.W. O'Hara. 2004. Root-nodule bacteria of indigenous legumes in the northwest of Western Australia and their interaction with exotic legumes. Soil Biol. Biochem. 36: 1319-1329. 